\title{
Relevant Factors Affecting the Clinical implementation Competence of Dental Hygienists
}

\author{
Su jin Lee , Jong Hwa Yum and Hye Jin Kim ${ }^{\dagger}$ \\ Department of Biomedical Health Science, Dong-Eui University, Busan, Korea \\ khj1126@deu.ac.kr
}

\begin{abstract}
This study was a descriptive correlation study to increase understanding of, and relationship among job satisfaction, critical thinking disposition and clinical competence of dental hygienists. Survey were undertake by dental hygienists working at the dental clinics located in B City from May 2012 to January 2013. A total of 176 questionnaires, excluding 24 incomplete ones, were analyzed. The influencing factors on clinical competence of dental hygieneists, it appeared that there were positive correlations between critical thinking, job satisfaction. It appeared that the higher the critical thinking, the higher the decision making ability, and the higher the job satisfaction, the more those factors affected the clinical competence. The result of this study indicate confirm that critical thinking and job satisfaction should be considered in order to improve the clinical competence of dental hygieneists, This study suggests an attempt at intergrated education for the qualitative improvement of clinical dental hygiene services and the revitalization of such a program.
\end{abstract}

Keywords: Critical Thinking, Job Satisfaction, Dental Hygienist

\section{Introduction}

With changes to the internal and external dental and medical environment, such as the development of dentistry technology, improvement of people's dental knowledge, and changes in their desire for oral health, there is a need for high-level specialization and qualitative improvement of subdivided dental care services [1]. Highly professional clinical competence by dental hygienists is required for the qualitative improvement of dental care services. Therefore, in clinical practice, dental hygienists need to judge patients' conditions correctly, predicting and coping with problems that may occur. High-level job performance capabilities are required by dental hygienists, who are the resources for creating knowledge and value in the field of oral health services [2].

In order to increase the work ability of dental hygienists, it is necessary to establish a suitable dental hygiene plan that is based on the analysis results of the individual characteristics required to perform the position. As the integrated clinical dental hygiene education is being operated in Korea's dental hygiene education course, the integrated clinical practice capacity that can be applied in the field involves raising students' clinical judgment abilities, decision making abilities, and problem solving abilities [3].

This study aims to assist in the improvement of dental hygienists' clinical competence by identifying the relevant factors, taking into consideration autonomy, and analyzing the factors that affect their clinical competence. 


\section{Subjects and Method}

\subsection{Subjects}

Surveys were undertaken by dental hygienists working at the dental clinics located in B City from May 2012 to January 2013. A total of 176 questionnaires, excluding 24 incomplete ones, were analyzed.

\subsection{Methods}

\subsubsection{Clinical Competence}

The clinical competence tool developed by Won-hee Lee et al [4]. and the modification by Hyun-young Choi was used [5]. A higher score represented a higher clinical competence

\subsubsection{Critical Thinking}

This involved an individual's ability to make judgments for himself or herself in order to undertake problem solving and decision making within personal and professional work. The California Critical Thinking Disposition Inventory (CCTDI), which was modified and supplemented by Ji-won Hwang [6] was used and a higher score represented a higher critical thinking disposition.

\subsubsection{Job Satisfaction}

This involved the favorable attitude toward his or her job, and the positive psychological state that occurred with different job characteristics and clear work requirements. This study used the tool developed by Slavitt et al [7]. that was modified and supplemented by Geumhye Won [8] A higher score represented higher job satisfaction.

\subsubsection{Decision Making Ability}

We used the tool for clinical decision making ability developed by Jenkins [9], adapted and modified by Baek [10] and then modified by Kim and Jang] as the content appropriate for the survey of dental hygienists. A higher measured score represents a higher clinical decision making ability.

\subsubsection{Autonomy}

Autonomy pertains to the independence observed within the job's characteristics or work activities. This study used the tool developed by Schutzenhofer [11] and adapted by Han et. al. [12]. A higher measured score represented a higher autonomy.

\subsection{Statistical Analysis}

The questionnaire's scores were statistically analyzed using SPSS Statistics 2.0 and the level of significance was 0.05 . The general subject characteristics were presented as a number and a percentage. The differences in critical thinking disposition, clinical competence, job satisfaction, decision making ability, and autonomy according to the subjects' general characteristics were analyzed using t-tests and ANOVA. Pearson's correlation coefficient was used to analyze the relevance between clinical competence, critical thinking, job satisfaction, and autonomy. Multiple regression analysis was used to analyze the affecting factors. 


\section{Results}

\subsection{Clinical Competence, Critical Thinking, Job Satisfaction, Decision Making Ability, and Autonomy According to the General Characteristics}

In the area of clinical competence, the highest levels in the dental hygienists' general

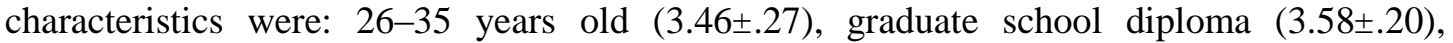

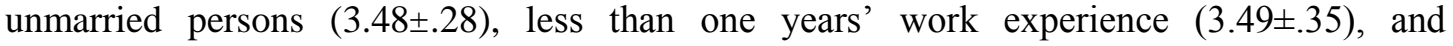
consulting room for the work department $(3.47 \pm .27)$. In the area of clinical thinking the general characteristics were highest for those: over 36 in age (3.22 \pm .24$)$, with a university

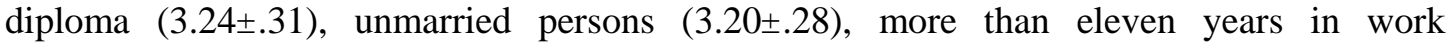
experience (3.24 \pm .24$)$, and reception as the work department (3.22 \pm .25$)$. In the area of job satisfaction these characteristics were highest: under 25 years old (3.07 \pm .30$)$, graduate school diploma (3.17 \pm .29$)$, unmarried persons (3.06 \pm .27$)$, less than one year of work experience

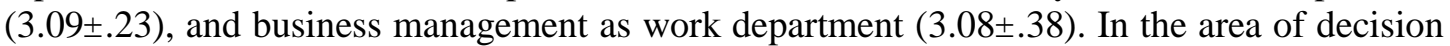
making ability, these characteristics were highest: over 36 years old (3.26 \pm .20$)$, university diploma (3.36 \pm .30$)$, unmarried persons (3.33 \pm .28$)$, less than one year of work experience

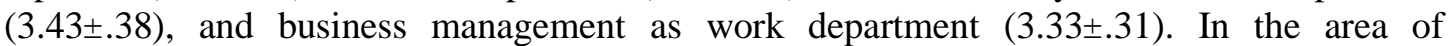

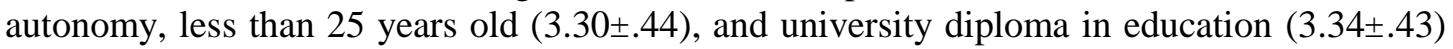
were statistically significant $(\mathrm{p}=.02)$

\subsection{Correlation between Clinical Competence and Critical Thinking, Job Satisfaction, Decision Making Ability, and Autonomy}

The correlation between clinical competence and critical thinking, job satisfaction, decision making ability, and autonomy of dental hygienists showed a positive correlation in critical thinking $(\mathrm{r}=.467, \mathrm{p}=.000)$, job satisfaction $(\mathrm{r}=.312, \mathrm{p}=.000)$, decision making ability $(\mathrm{r}=.459, \mathrm{p}=.000)$, and autonomy $(\mathrm{r}=.409, \mathrm{p}=.000)$.

\subsection{Factors Affecting Clinical Competence}

As a result of conducting multiple regression to investigate the factors affecting clinical competence, multicollinearity ( VIF $\leq 10$ ) and autocorrelation (DW 2.082) were conducted and the regression model was found to be suitable. As a result of the analysis, the higher the critical thinking about clinical competence $(\beta=.325, \mathrm{p}=.000)$, the higher the decision making ability $(\beta=.258, \mathrm{p}=.001)$, while the higher the job satisfaction $(\beta=.167, \mathrm{p}=.008)$, the more significant the effect. The degree of explanatory power was 36.8 .

\section{Discussion}

The clinical competence of dental hygienists is an essential requirement for dental hygienists to provide patients with a safe and qualitative dental hygiene process, and its evaluation is very important as it is closely related to the efficient human resource management of dental clinics

The mean clinical competence of this study's subjects was $3.45 \pm .46$, mean critical thinking $3.18 \pm .26$, mean job satisfaction $3.04 \pm .29$, mean decision making ability $3.31 \pm$ .27 , and mean autonomy $3.24 \pm .37$, showing somewhat different results compared to the results of the study on the clinical competence (2.77) and critical thinking (4.10) conducted by Ji-won Hwang[6], and lower values than the study regarding critical thinking (3.60), clinical decision making ability (3.42), and job satisfaction (3.12) conducted by Sam-chul [13] Jung his is interpreted to mean that exists a greater need for professionalism by nurses compared to the oral health workforce's job classifications, and that differences may be dependent on the field of work and workload. 
The difference in clinical competence according to the general characteristics was high for 26-35 years old, higher education, work experience of less than one year and more than eleven years, and consulting room work; however, there was no significant difference. In Jina Park's[14] study, with participants who were older than 36 years, there were statistically significant differences in the clinical competence scores of the groups who had clinical experience of less than 5-10 years and more than 10 years compared to other groups. Mi-hye Sung and Ok-bong Um [15] reported that there were significant differences in role, age, clinical career, current position, and department. As the acquisition of knowledge and technology is an important element in improving clinical competence, the improvement of the working environment in dental clinics and the conditions for increasing satisfaction of work will need to be provided. The results for critical thinking according to the general characteristics were not significant. The higher the education and the shorter the work experience, the higher the job satisfaction, and unmarried hygienists showed higher satisfaction levels; however, there was no significant difference. There was a significant difference in decision making ability depending on the period of work experience. It was shown that the shorter the period of work experience, the higher the decision making ability. This is thought to be due to the fact that more experienced hygienists often need to play perform the tasks of patient counseling, hospital management, and personnel management in clinical scene while less experienced hygienists need diverse and fast coping capabilities in the area of treatment services for patients. There was a significant difference in autonomy as the university graduates showed higher autonomy than the three-year college graduates or graduate school graduates. Many of the highly educated dental hygienists working in the clinical scene will be engaged in research or focus on self-development, while the dental hygienists with education lower than university levels are thought to display more autonomy in the clinical environment if their clinical experience is estimated to be less than five years

As a result of investigating the correlation between clinical competence of dental hygienists and associated factors, there was a positive correlation in critical thinking $(\mathrm{r}=.467$, $\mathrm{p}=.000)$, job satisfaction $(\mathrm{r}=.312, \mathrm{p}=.000)$, decision making ability $(\mathrm{r}=.459, \mathrm{p}=.000)$, and autonomy $(\mathrm{r}=.409, \mathrm{p}=.000)$. According to the previous studies, critical thinking, decision making ability, and job satisfaction in the study by Sam-chul Jung [13], critical thinking and clinical competence in the study by Jin-a Park[14], and critical thinking, job satisfaction, and clinical competence in the study by Mi-hye Sung and Ok-bong Um [15] appeared to have correlations, showing results similar to this study.

It was demonstrated that that the higher the critical thinking, the higher the decision making ability; and the higher the job satisfaction, the more significant the effect of the factors affecting clinical competence would be. Jin-ju Yang reported that clinical competence and critical thinking have a significant effect while Seung-gyo Jung reported that subjects with high critical thinking and problem solving abilities have high clinical competence[16].

Overall, critical thinking, job satisfaction, decision making ability, and autonomy of dental hygienists, which are the factors affecting the clinical competence of dental hygienists, were similar in level to other professionals, and it could be seen that the higher these factors, the higher the clinical competence. However, there are some limitations in conducting studies when they are directly compared with the previous studies because there are insufficient studies about the effects on clinical competence for the dental hygienists. It is thought that the studies to increase the clinical competence of dental hygienists and in-depth studies on the factors that affect it are needed, and the studies and education on the various factors that can increase the professional and efficient clinical competence of dental hygienists need to be made. 


\section{Conclusion}

As a result of analyzing clinical competence, critical thinking, job satisfaction, decision making ability, and autonomy according to the general characteristics, the score of university and college graduates was higher than that of graduate school graduates $(\mathrm{p}=.02)$, and the score was higher $(\mathrm{p}=.05)$ as the work experience was less in decision making ability, showing a significant difference.

As a result of investigating the relevance between clinical competence and critical thinking, job satisfaction, decision making ability, and autonomy, it appeared that there were positive correlations between critical thinking $(\mathrm{r}=.467, \mathrm{p}=.000)$, job satisfaction $(\mathrm{r}=.312, \mathrm{p}$ $=.000)$, decision making ability $(\mathrm{r}=.459, \mathrm{p}=.000)$, and autonomy $(\mathrm{r}=.409, \mathrm{p}=.000)$.

It appeared that the higher the critical thinking $(\beta=.325, \mathrm{p}=.000)$, the higher the decision making ability $(\beta=.258, p=.001)$; and the higher the job satisfaction $(\beta=.167, p=.008)$, the more those factors affected the clinical competence.

Factors such as critical thinking, job satisfaction, decision making ability, and autonomy should be considered in order to improve the clinical competence of dental hygienists. This study suggests an attempt at integrated education for the qualitative improvement of clinical dental hygiene services and the revitalization of such a program.

\section{References}

[1] K. S. Kim and M. S. Yu, The Journal of Dental Hygiene Science, vol. 4, (2004), p. 3.

[2] Y. S. Cho, The Journal of Nam Seoul University, vol. 10, (2004), p. 409.

[3] Y. S, Cho, H. S. Bae and S. Y. Lee, Clinical Dental Hygiene Care, Namseoul University, Seoul (2013).

[4] W. H. Lee, Yonsei University, Seoul (2004).

[5] H. Y. Choi, Chosun University, Seoul (1992).

[6] J. W. Hwang, Ewha Women University, Seoul (1998).

[7] D. B. Slavitt, Nursing Research, vol. 27, p. 114 (1978).

[8] G. H. Won, Daejeon University, Daejeon (2003).

[9] H. M. Jenkins, Journal of Professional Nursing, vol. 1, (1985), p. 221.

[10] M. K. Baek, Yonsei University, Seoul (2004).

[11] K. K. Schutzenhofer, Journal of Psychological Nursing and Mental Health Services, vol. 21, (1983), p. 25.

[12] K. J. Han, E. O. Lee, S. A. Park, Y. S. Ha and G. S. Kim, Seoul University Publisher, Seoul (1994).

[13] S. C. Jeong and D. Y. Jeong, Journal of Korean Academy of Nursing Administration, vol. 17, (2011), p. 443.

[14] J. A. Park, Ulsan University, Ulsan (2008).

[15] M. H. Sung and O. B. Eum, The Korean Journal of Fundamentals of Nursing, vol. 10, (2010), p. 274.

[16] S. J. Lee, J. H. Yum and H. J. Kim, Advanced Science and Technology Letters, vol. 33, (2013), p. 30.

\section{Authors}

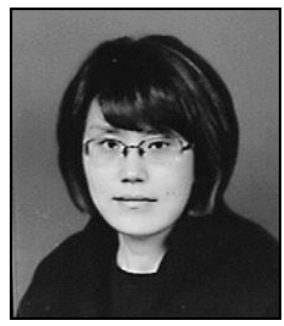

\section{Hye Jin Kim}

19 Feb. 2004: Ph.D. degree at Keimyung University in Korea. 3 Mar. 2010 - 2 Feb. 2012: assistant professor Baekseok University in Korea

1 Mar. 2012 - recent: assistant professor Dong-eui University in Korea. 


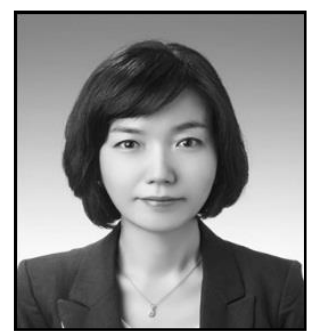

\section{Su Jin Lee}

19 Feb. 2010: M.P.H degree at Ko-sin University in Korea

1 Mar 2013 - recent: Department of Biomedical Health Science, Dong-eui University in Korea.

Research Areas: The relationship between the degree of autonomy and decision-making ability of clinical dental hygienists

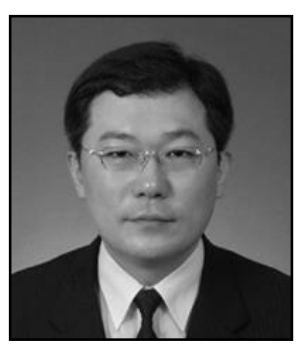

\section{Jong Hwa Yum}

1 Feb. 1990 - 6 Aug. 1994: Department of Bacteriology at National Institute of Health (NIH) in Korea.

19 Feb. 1998: Ph.D. degree at Dankuk University in Korea.

1 Mar. 2008 - recent: assistant professor Dong-eui University in Korea. 